\title{
Kainate Seizures Cause Acute Dendritic Injury and Actin Depolymerization In Vivo
}

\author{
Ling-Hui Zeng, ${ }^{\star}$ Lin Xu, ${ }^{\star}$ Nicholas R. Rensing, Philip M. Sinatra, Steven M. Rothman, and Michael Wong \\ Department of Neurology and the Hope Center for Neurological Disorders, Washington University School of Medicine, St. Louis, Missouri 63110
}

\begin{abstract}
Seizures may cause brain injury via a variety of mechanisms, potentially contributing to cognitive deficits in epilepsy patients. Although seizures induce neuronal death in some situations, they may also have "nonlethal" pathophysiological effects on neuronal structure and function, such as modifying dendritic morphology. Previous studies involving conventional fixed tissue analysis have demonstrated a chronic loss of dendritic spines after seizures in animal models and human tissue. More recently, in vivo time-lapse imaging methods have been used to monitor acute changes in spines directly during seizures, but documented spine loss only under severe conditions. Here, we examined effects of secondary generalized seizures induced by kainate, on dendritic structure of neocortical neurons using multiphoton imaging in live mice in vivo and investigated molecular mechanisms mediating these structural changes. Higher-stage kainate-induced seizures caused dramatic dendritic beading and loss of spines within minutes, in the absence of neuronal death or changes in systemic oxygenation. Although the dendritic beading improved rapidly after the seizures, the spine loss recovered only partially over a $24 \mathrm{~h}$ period. Kainate seizures also resulted in activation of the actin-depolymerizing factor, cofilin, and a corresponding decrease in filamentous actin, indicating that depolymerization of actin may mediate the morphological dendritic changes. Finally, an inhibitor of the calcium-dependent phosphatase, calcineurin, antagonized the effects of seizures on cofilin activation and spine morphology. These dramatic in vivo findings demonstrate that seizures produce acute dendritic injury in neocortical neurons via calcineurindependent regulation of the actin cytoskeleton, suggesting novel therapeutic targets for preventing seizure-induced brain injury.
\end{abstract}

Key words: epilepsy; seizure; dendrite; kainic acid; cofilin; calcineurin

\section{Introduction}

Seizures may cause brain injury via a number of mechanisms, potentially contributing to neurological and cognitive deficits in epilepsy patients. Although seizures can induce neuronal death in some situations, they may also have "nonlethal" pathophysiological effects on neuronal structure and function. Dendritic spines represent the structural sites of contact for the majority of excitatory, glutamatergic synaptic inputs onto cortical neurons and are strongly implicated in mechanisms of synaptic plasticity and learning. A variety of studies demonstrate a loss of dendritic spines in pathological specimens from animal seizure models (Olney et al., 1983; Muller et al., 1993; Drakew et al., 1996; Isokawa, 1998; Jiang et al., 1998) or human epilepsy patients (Scheibel et al., 1974; Isokawa and Levesque, 1991; Multani et al., 1994), suggesting that seizures can cause dendritic injury. However, these previous studies using conventional histological analysis of fixed tissue are somewhat limited by the difficulty in distinguishing direct effects of seizures from potential confounding

Received March 5, 2007; revised Aug. 31, 2007; accepted Sept. 9, 2007.

This work was supported by National Institutes of Health (NIH) Grants K02 NS045583 and R01 NS056872 (M.W.), R01 NS42936 and R21 NS045652 (S.M.R.), NIH Neuroscience Blueprint Core Grant NS057105 (Washington University), and by the Alafi Family Foundation.

*L.-H.Z. and L.X. contributed equally to this work.

Correspondence should be addressed to Dr. Michael Wong, Department of Neurology, Box 8111, Washington University School of Medicine, 660 South Euclid Avenue, St. Louis, M0 63110. E-mail:wong_m@wustl.edu. DOI:10.1523/JNEUROSCI.0983-07.2007

Copyright $\odot 2007$ Society for Neuroscience 0270-6474/07/2711604-10\$15.00/0 or coincidental factors and by the relatively slow time course of analysis, typically spanning hours to days.

Compared with conventional fixed tissue studies, advances in cellular imaging techniques now allow repetitive, time-lapse imaging of dendritic spines within the living brain in vivo (Lendvai et al., 2000; Grutzendler et al., 2002; Trachtenberg et al., 2002; Holtmaat et al., 2005), so that the same dendrites can be followed before and after seizures to more directly assess the effects of seizures (Mizrahi et al., 2004; Rensing et al., 2005). Furthermore, because dendritic spines have been found by these newer methods to have a previously unanticipated degree of motility with a time course of seconds to minutes, in vivo time-lapse imaging can also study acute immediate effects of seizures on a much faster time scale. Two recent studies have used these methods in selected animal seizure models and found some evidence of dendritic injury, but the effects were relatively mild or seen only under extreme conditions (Mizrahi et al., 2004; Rensing et al., 2005). In the present in vivo imaging study, we demonstrate a more robust, acute dendritic effect of seizures induced by a different model, systemic administration of kainate. Furthermore, because physiological activity has been shown to affect dendritic function and structure by modulating actin networks (Kim and Lisman, 1999; Krucker et al., 2000; Fukazawa et al., 2003; Okamoto et al., 2004; Lin et al., 2005; Ouyang et al., 2005; Kramar et al., 2006), we also show that these acute morphological effects of seizures on dendrites are directly related to changes in the poly- 
merization state of actin mediated by the calcium-dependent phosphatase, calcineurin.

\section{Materials and Methods}

Animals and reagents. Two- to three-month-old transgenic mice with a C57BL/6 background expressing enhanced green fluorescent protein (GFP) under a thy1 promoter (line GFP-M) (Feng et al., 2000) were used for all in vivo imaging experiments. In neocortex, the GFP-M mice exhibit expression of GFP in a subpopulation of pyramidal neurons, primarily in cortical layer 5 and, to a lesser extent, layer $2 / 3$. Two- to threemonth-old C57BL/6 wild-type mice were used for separate experiments for rhodamine-phalloidin and Fluoro-Jade B labeling and Western blot analysis for actin and cofilin. Care and use of animals conformed to a protocol approved by the Washington University School of Medicine Animal Studies Committee.

Rhodamine-phalloidin was obtained from Molecular Probes (Eugene, OR). Anti-cofilin antibody and anti-phospho-cofilin (Ser3) antibody were obtained from Cytoskeleton (Denver, CO) and Cell Signaling (Beverly, MA), respectively. Fluoro-Jade B was obtained from Chemicon (Temecula, CA). Kainate, anti-MAP2, and anti-PSD95 antibodies were purchased from Sigma (St. Louis, MO). FK506 was purchased from LC Laboratories (Woburn, MA). FK506 was initially dissolved in 100\% ethanol at $10 \mathrm{mg} / \mathrm{ml}$, stored at $-20^{\circ} \mathrm{C}$, and diluted with a solution of $5 \%$ Tween 80 and 5\% PEG 400 immediately before injection.

In vivo imaging. Animal surgery, image acquisition, and image analysis were performed by similar methods as previously reported (Rensing et al., 2005). Briefly, GFP-M mice were anesthetized with isoflurane anesthesia and held in a custom-made stereotaxic device, which could be mounted to the microscope stage. A heating pad and lamp were used to maintain body temperature while under anesthesia. A rectangular cranial window $(\sim 2.5 \times 2 \mathrm{~mm})$ was first drilled in the skull with the center of the window $\sim 3 \mathrm{~mm}$ posterior to bregma and $2 \mathrm{~mm}$ lateral to midline. A glass coverslip $(\# 1.5,8 \mathrm{~mm})$ was centered over the cranial window and attached to the skull with dental acrylic.

Images of dendrites and dendritic spines of neocortical neurons expressing GFP were obtained through the cranial window with a multiphoton microscope (LSM 510; Zeiss, Thornwood, NY) and a waterimmersion objective [Zeiss, $40 \times, 0.8$ numerical aperture (NA), infraredadjusted]. A titanium-sapphire pulsed infrared laser (Coherent, Santa Clara, CA) was used to stimulate GFP at $900 \mathrm{~nm}$. Low power images $\sim 50-100 \mu \mathrm{m}$ below the neocortical surface were first obtained to identify regions with GFP-expressing dendrites. At higher magnification $(5 \times$ digital zoom), $Z$-stacks of $6-10$ images separated by $1 \mu \mathrm{m}$ steps were taken of dendrites and accompanying spines. Individual images were acquired at 12 bits with frame averaging (2-4 times).

After obtaining control images under anesthesia, mice were injected with kainate $(30 \mathrm{mg} / \mathrm{kg}$, i.p.) or saline and allowed to recover from anesthesia. In kainate-injected mice, typical progression through different stages of clinical seizure activity occurred and were graded according to a modified Racine scale (Racine, 1972): stage 1, behavioral arrest with mouth/facial movements; stage 2, head nodding; stage 3, forelimb clonus; stage 4, rearing; stage 5 , rearing and falling; stage 6 , loss of posture and generalized convulsive activity. With the dose of kainate used (30 $\mathrm{mg} / \mathrm{kg}$ ), we found that some mice progress to stage 4 but do not progress further, whereas most mice transition to stage 5 within $\sim 20-30 \mathrm{~min}$. Before terminating the seizures and reanesthetizing the mice for reimaging, mice that progressed from stage 4 to stage 5 were allowed to remain in stage 5 for $30 \mathrm{~min}$, whereas mice that did not progress further than stage 4 within 30 min were allowed to stay in stage 4 for an additional 30 $\min (\sim 60 \mathrm{~min}$ of total seizures in both cases). Using blood vessel landmarks as references, the same dendrites from the control period were reimaged at intervals of $0,1,2$, and, in some cases, 4 and $24 \mathrm{~h}$ after termination of the seizures. To test for the effects of calcineurin inhibition on seizure-induced dendritic changes, additional groups of mice were injected with FK-506 (2.5 mg/kg, i.p.) either $2 \mathrm{~h}$ before kainate (30 $\mathrm{mg} / \mathrm{kg}$, i.p.) or immediately after termination of the kainate-induced seizures. To control for the potential direct toxic effects of kainate on dendrites, other mice were injected with pentobarbital (30 mg/kg, i.p.) 30 min before kainate $(30 \mathrm{mg} / \mathrm{kg}$, i.p.) to suppress seizure activity.
Post hoc image analysis was performed using MetaMorph software (Molecular Devices, Downingtown, PA) to evaluate changes in the number of dendritic spines over time, as described previously (Rensing et al., 2005). Individual images in $Z$-stacks were first projected on to a single plane to facilitate spine counting in the $x-y$ plane. Spines were operationally defined as perpendicular projections out of the main axis of the dendrite that were narrower than the dendrite from which they arose and could progressively taper, maintain their width, or form "caps." All readily resolvable spines in the initial image of a sequence were tagged and then all tags were transferred to each subsequent image in the time series for comparison. In addition to spine counting, a qualitative scoring system was also used to grade the degree of beading that frequently occurred after seizures: no beading; mild beading (visible beads with diameter of beads $<3 \times$ the diameter of the original dendrite with normal intervening segments of dendrite); severe beading (visible beading with diameter of beads $\geq 3 \times$ the diameter of the original dendrite without normal intervening segments of dendrite. Two different people analyzed the imaging data independently to confirm interobserver reliability of the analysis method.

Video-EEG recording. In separate experiments, video-EEG recordings were performed to characterize the behavioral-electrographic correlate of kainate-induced seizures in more detail. Under isoflurane anesthesia, mice had surgical implantation of right and left frontal epidural screw electrodes ( $\sim 1 \mathrm{~mm}$ posterior to bregma and $1 \mathrm{~mm}$ lateral to midline), a midline occipital reference screw electrode $(\sim 1 \mathrm{~mm}$ posterior to lambda), and an insulated silver wire electrode inserted stereotaxically into the right hippocampus ( $2 \mathrm{~mm}$ posterior to bregma, $1.5 \mathrm{~mm}$ lateral to midline, $1 \mathrm{~mm}$ deep). After at least $24 \mathrm{~h}$ after recovery from surgery, mice were injected with $30 \mathrm{mg} / \mathrm{kg}$ kainate intraperitoneally and then monitored by video-EEG. EEG signals were amplified and filtered $(1-100 \mathrm{~Hz})$ using standard AC amplifiers (Grass P-511; Astro-Med, West Warwick, $\mathrm{RI}$ ) and digitized with commercial hardware and software (Axon Digidata 1322 and Axoscope; Molecular Devices) on a personal computer. Time-locked video data were recorded using a Sanyo Day-Night camera and a Darim MG-100 MPEG video capture card (Darim Vision, Pleasanton, CA).

F-actin and Fluoro-Jade B labeling. The rhodamine-phalloidin labeling method was used, as described previously (Ouyang et al., 2005, 2007), to measure F-actin levels in wild-type C57BL/6 mice after saline or kainate $(30 \mathrm{mg} / \mathrm{kg}$, i.p.) injection. Phalloidin has a high affinity for F-actin and is selectively concentrated in dendritic spines of neurons (Capani et al., 2001). After at least $30 \mathrm{~min}$ of stage 5 seizure activity, mice were perfusion fixed with $4 \%$ paraformaldehyde. Coronal brain sections $(50 \mu \mathrm{m})$ were subsequently cut with a vibratome. Sections were treated with $0.7 \%$ Triton X-100 in 10 mм PBS, pH 7.2, for $1 \mathrm{~h}$, blocked with $5 \%$ serum for $1 \mathrm{~h}$, and then incubated with rhodamine-phalloidin $(1: 200)$ overnight at $4^{\circ} \mathrm{C}$. Sections were washed three times and mounted with anti-fade medium for confocal imaging. Images of the stratum radiatum of CAl regions of hippocampus and layer 1-3 of neocortex were acquired with a Zeiss LSM PASCAL confocal microscope. A high-power objective $(63 \times, 1.2 \mathrm{NA})$ was used to confirm the punctate labeling typical of spines (Ouyang et al., $2005,2007)$, and a low-power objective $(25 \times, 0.8 \mathrm{NA})$ was used to obtain images for regional F-actin intensity measurements. Regions of interest from images were selected within the striatum radiatum of CA1 and layer $1 / 2$ of neocortex to measure the average brightness of F-actin labeling using MetaMorph analysis software. Double-labeling experiments involving immunolabeling of MAP2 and PSD95 with F-actin staining were performed to confirm the primary dendritic localization of F-actin in these studies. On separate sections, labeling for Fluoro-Jade B or terminal deoxynucleotidyl transferase-mediated biotinylated UTP nick end labeling (TUNEL) (Chemicon) was performed using kit instructions and previously published methods (Schmued and Hopkins, 2000; Wong et al., 2003).

Western blotting. For Western blot analysis of cofilin and actin, the brains were removed from C57BL/6 wild-type mice at various times after saline or kainate $(30 \mathrm{mg} / \mathrm{kg}$, i.p.) injection. In some experiments testing the effects of a calcineurin inhibitor, FK506 $(2.5 \mathrm{mg} / \mathrm{kg}$, i.p. $)$ was injected $2 \mathrm{~h}$ before kainate. The neocortex and hippocampi were dissected out and sonicated individually in SDS-PAGE sample buffer containing 3\% 
SDS, $2 \% \beta$-mercaptoethanol, and $5 \%$ glycerol in $60 \mathrm{~mm}$ Tris buffer, $\mathrm{pH}$ 6.7, as described previously (Ouyang et al., 2005, 2007). Samples were boiled for $5 \mathrm{~min}$ and stored at $-20^{\circ} \mathrm{C}$. Protein concentration was determined with the Lowry method. Thirty micrograms of protein were separated by $15 \%$ SDS-PAGE and transferred to polyvinylidene difluoride membranes. After incubation with a primary antibody (1:1000; Cell Signaling) that recognized phosphorylated cofilin (p-cofilin) at Ser3, the membranes were labeled with peroxidaseconjugated secondary antibody and visualized by ECL detection kit (Pierce, Rockford, IL). The blots were reprobed for total cofilin (1:1000) and $\beta$-actin (1:4000). The signals were scanned for quantitative analysis with ImageJ.

Measurements of the F-actin to G-actin ratio were also made by Western blotting, similar to previously published methods (Gu et al., 2006). Cortex was isolated and homogenized in cold lysis buffer $\left(10 \mathrm{mM} \mathrm{K}_{2} \mathrm{HPO}_{4}, 100 \mathrm{~mm} \mathrm{NaF}, 50\right.$ mм KCl, $2 \mathrm{~mm} \mathrm{MgCl}_{2}$, 1 mм EGTA, $0.2 \mathrm{~mm}$ dithiothreitol, $0.5 \%$ Triton X-100, $1 \mathrm{~m}$ sucrose, $\mathrm{pH} 7.0)$ and then centrifuged at $15,000 \times g$ for $30 \mathrm{~min}$. The supernatant was used for measurement of soluble actin (G-actin). To measure F-actin, the pellets were resuspended in lysis buffer plus an equal volume of $1.5 \mathrm{~m}$ guanidine hydrochloride, $1 \mathrm{~m}$ sodium acetate, $1 \mathrm{~mm}$ $\mathrm{CaCl}_{2}, 1 \mathrm{~mm}$ ATP, and $20 \mathrm{~mm}$ Tris-HCl, $\mathrm{pH} 7.5$, and incubated on ice for $1 \mathrm{~h}$ to depolymerize F-actin, with gentle mixing every $15 \mathrm{~min}$. The samples were centrifuged at $15,000 \times g$ for 30 $\mathrm{min}$, and this supernatant was also used to measure actin (as a reflection of insoluble F-actin). Samples from the supernatant (G-actin) and pellet (F-actin) fractions were proportionally loaded and analyzed by Western blotting.

Arterial blood gas analysis. To assess the potential effects of kainate seizures on systemic variables, arterial blood gases were monitored immediately after $30 \mathrm{~min}$ of stage 5 kainate seizure activity. In separate mice from the imaging studies, blood samples from femoral artery catheterization were obtained under anesthesia, and $\mathrm{pH}$ and $\mathrm{pO}_{2}$ were measured using a CIBA-Corning 238 pH/Blood Gas Analyzer.

Statistics. One-way ANOVA with Tukey-Kramer posttests for multiple comparisons was used to compare changes in dendritic spine number, F-actin intensity, and quantified protein expression between different treatment groups. Chi-square test of independence was used to compare the distribution of dendritic beading severity as a function of seizure stage. All data are expressed as mean \pm SEM. Statistical significance was defined as $p<0.05$.

\section{Results}

Kainate seizures activate neocortical neurons in a seizurestage dependent manner but do not cause neuronal death or systemic perturbations in $\mathrm{C} 57 \mathrm{BL} / 6$ mice

We chose to study the acute effects of kainate-induced seizures on dendritic spines, because previous in vivo imaging studies found only modest effects of other seizure models on dendrites (Mizrahi et al., 2004; Rensing et al., 2005), whereas acute kainate-induced seizures directly activate glutamate receptors, which may be more relevant to the phenomenon under study (Ben-Ari and Cossart, 2000). In addition, we imaged neurons specifically in neocortex to assess the effect of (secondary) generalized seizure activity, which may have more Scale bars, $200 \mu \mathrm{m}$.
B C57BL6 mice, Kainate $30 \mathrm{mg} / \mathrm{kg}$

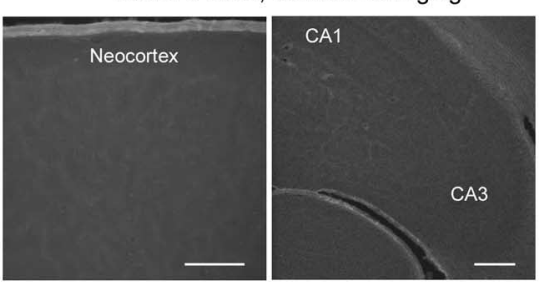

C57BL6 mice, Kainate $45 \mathrm{mg} / \mathrm{kg}$

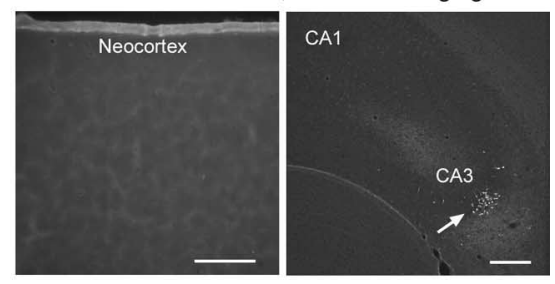

SD rats, Kainate $15 \mathrm{mg} / \mathrm{kg}$

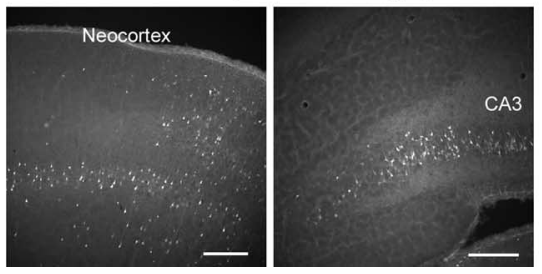

Figure 1. Staged electrographic seizures and limited neuronal death in the kainate model. $\boldsymbol{A}$, Bilateral cortical and hippocampal EEG recordings demonstrate the evolution of electrographic ictal discharges correlating with different clinical stages of seizures

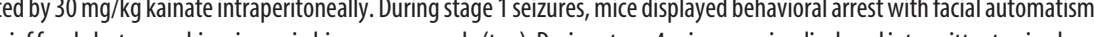

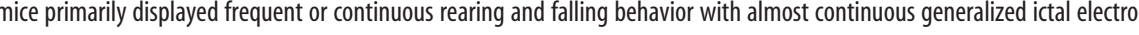
resulted in no detectable cell death in C57BL/6 mice, as assayed by Fluoro-Jade B staining $3 \mathrm{~d}$ after kainate-induced seizures (top). arrow) but not in CA1, dentate gyrus, or neocortex of C57BL/6 mice (middle). By comparison, kainate seizures caused by 15 $\mathrm{mg} / \mathrm{kg}$ produced widespread Fluoro-Jade B staining in both neocortex and hippocampus of Sprague Dawley (SD) rats (bottom).

widespread, robust effects than the previously examined focal seizures (Mizrahi et al., 2004; Rensing et al., 2005).

Although the behavioral, electrophysiological, and histological correlates of the kainate seizure model have been described extensively (Ben-Ari and Cossart, 2000; Leite et al., 2002), we performed video-EEG recordings to confirm the behavioralelectrographic features of kainate seizures and allow direct correlation with structural changes observed in the imaging studies. After intraperitoneal injection of $30 \mathrm{mg} / \mathrm{kg}$ kainate, mice $(n=8)$ displayed a stereotypical progression of clinical seizure behavior that evolved through different stages over 30-60 min. In stage 1 and 2, mice predominantly exhibit behavioral arrest/freezing with subtle facial automatisms and head nodding, which correlated with focal ictal electrographic discharges in hippocampus on EEG with minimal spread to neocortical electrodes (Fig. $1 \mathrm{~A}$, top). With higher-stage seizures, mice displayed progressively more severe bilateral motor manifestations, including bilateral forelimb clonus (stage 3), rearing (stage 4), and rearing and falling (stage 5), which were correlated with bilateral ictal electrographic discharges in neocortex, reflecting secondary generalization of the initial seizures from the hippocampus (Fig. $1 A$, middle and bottom). As seizures progressed from stage 4 to stage 5 , the EEG pattern gradually transitioned from intermittent electrographic seizures to almost continuous bilateral discharges (ictal 
discharges occupying $46 \pm 11 \%$ of the EEG during stage 4 and $92 \pm 6 \%$ during stage $5 ; n=5$ mice). These findings indicate that higher-stage kainate seizures extensively activate neocortical neurons in a dose (i.e., seizure stage)-dependent manner, which is directly relevant to the accompanying imaging studies in neocortex.

Of specific relevance to the imaging studies, despite using the same concentration of kainate (30 mg/kg i.p.), some mice never progressed from stage 4 to stage 5 within the temporal constraints used for the imaging studies, whereas others progressed into stage 5. Of the mice that progressed to stage 5, frequent rearing and falling was the predominant clinical feature, with only a couple mice also displaying rare, brief episodes of loss of posture and severe convulsive motor activity (stage 6) during the $30 \mathrm{~min}$ period.

Because kainate seizures may trigger neuronal death and we are primarily interested in studying "nonlethal" mechanisms of seizure-induced dendritic injury, we purposely took advantage of the fact that mice with a $\mathrm{C} 57 \mathrm{BL} / 6$ background have been reported to be relatively resistant to kainate excitotoxic neuronal death (Schauwecker and Steward, 1997). We confirmed the previous studies that kainate at a dose of $30 \mathrm{mg} / \mathrm{kg}$ activated no cell death after 4, 24, and $72 \mathrm{~h}$ in the hippocampus and neocortex of C57BL/6 mice ( $n=5)$, as assayed by Fluoro-Jade B staining (Fig. $1 \mathrm{~B}$, top). As a positive control, at higher kainate doses of 45 $\mathrm{mg} / \mathrm{kg}$, limited cell death was occasionally seen in the CA3 region of hippocampus only ( 2 of 4 mice), but not in CA1, dentate gyrus, or neocortex (Fig. $1 \mathrm{~B}$, middle). As a stronger positive control for the method, kainate $(15 \mathrm{mg} / \mathrm{kg})$ induced extensive cell death assayed by Fluoro-Jade B staining in hippocampus and neocortex in Sprague Dawley rats (Fig. $1 B$, bottom). Similarly, TUNEL staining revealed no evidence of neuronal death at 4,24 , and $72 \mathrm{~h}$ after kainate seizures in C57BL/6 mice, but extensive death in Sprague Dawley rats at $72 \mathrm{~h}$ (supplemental Fig. 1, available at www.jneurosci.org as supplemental material). These findings demonstrate that doses of kainate $(30 \mathrm{mg} / \mathrm{kg})$ used in the accompanying imaging studies do not cause neuronal death in C57BL/6 mice, and thus observed effects of kainate seizures on dendritic structure involve "nonlethal" mechanisms.

Given that systemic factors during seizures, such as hypoxemia or acidosis, could potentially cause dendritic changes independent of the electrical seizure activity, we also performed arterial blood gas analysis during kainate seizures. After $30 \mathrm{~min}$ of stage 5 seizure activity, there was no evidence of systemic hypoxemia or acidosis $\left(\mathrm{pO}_{2}=110.0 \pm 2.4 \mathrm{mmHg}, \mathrm{pH}=7.41 \pm 0.02\right.$; $n=8$ mice). These findings indicate that observed effects of kainate seizures on dendritic structure in the imaging studies are likely not secondary to perturbation of systemic factors.

\section{High-stage kainate seizures cause acute dendritic injury}

The effects of stage 4 and 5 kainate seizures on dendritic spines of neocortical neurons were assessed on GFP-M mice in vivo. Consistent with previous reports (Rensing et al., 2005), control mice injected with saline show minimal changes in dendritic spine number, with a $<5 \%$ change in spine number over a $>4 \mathrm{~h}$ period (Figs. 2, 3) ( $n=400$ total spines from 33 dendrites from 5 mice), and no signs of dendritic beading (Fig. 3). Stage 4 seizure activity usually also had minimal effects on dendritic spines, although overall there was a small but significant loss of spines, which was observed immediately after termination of the seizures and remained stable for the following $4 \mathrm{~h}$ (Fig. 2) $(n=596$ total spines from 47 dendrites from 7 mice). In most cases, stage 4 seizures

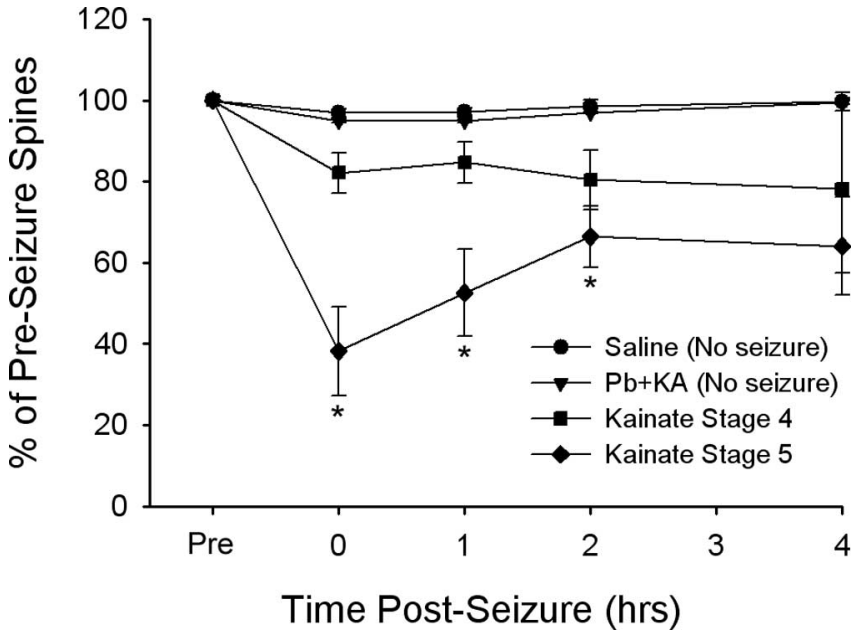

Figure 2. Kainate seizures cause an acute stage-dependent loss of dendritic spines, which is only partially reversible. Saline-injected mice demonstrated less than a $5 \%$ change in dendritic spines over a $4 \mathrm{~h}$ period ( $n=400$ total spines from 33 dendrites from 5 mice). Similarly, mice that were injected with kainate but had seizures suppressed by pentobarbital had no significant change in spines ( $\mathrm{Pb}+\mathrm{KA} ; n=198$ total spines from 17 dendrites from 2 mice). Stage 4 seizures induced a small but significant loss of spines, which was observed immediately after termination of the seizures and remained stable for the following $4 \mathrm{~h}(n=596$ total spines from 47 dendrites from 7 mice). In contrast, stage 5 seizures for 30 min resulted in a larger loss of dendritic spines, which only partially recovered over the $4 \mathrm{~h}$ period ( $n=531$ total spines from 49 dendrites from 9 mice). ${ }^{*} p<0.001,0.01$, and 0.05 at 0,1 , and $2 \mathrm{~h}$, respectively, by one-way ANOVA.

were not associated with any other gross morphological changes in dendrites, although in some cases $(\sim 15 \%)$, there was mild beading of the dendrites (Table 1). In contrast, stage 5 kainate seizures for $30 \mathrm{~min}$ typically caused very obvious morphological changes in dendrites and spines. Most dendrites ( $~ 80 \%)$ exhibited either mild or severe beading immediately after termination of the seizures, which almost totally resolved by $2-4 \mathrm{~h}$ (Fig. 3, Table 1). Along with the dendritic beading, Stage 5 seizures also resulted in an immediate loss of $>60 \%$ of dendritic spines (Figs. $2,3)(n=531$ total spines from 49 dendrites from 9 mice; $p<$ 0.001 by ANOVA compared with control and stage 4). Although there was some recovery of spines over the next $2 \mathrm{~h}$ that paralleled the resolution of the dendritic beading, a plateau in this recovery occurred between 2 and $4 \mathrm{~h}$ after the seizures, indicating a more persistent, longer-term loss of a subset ( $\sim 40 \%$ ) of spines (Fig. 2 ). In contrast to our previous studies with the focal 4-AP seizure model, in which a possible synergistic interaction of phototoxicity from the imaging method with the seizures was detected (Rensing et al., 2005), dendritic beading after kainate seizures was also seen in regions of neocortex outside of the original imaging fields (data not shown), indicating that the dendritic injury was a primary result of the kainate seizures independent of any contingent technical factors. In addition, as a control for possible direct toxic effects of kainate, mice injected with pentobarbital before kainate to suppress seizure activity had no signs of dendritic beading or loss of spines (Fig. 2) ( $n=198$ total spines from 17 dendrites from 2 mice).

Although previous fixed-tissue studies have documented chronic spine loss after seizures and the primary purpose of the present study was to document acute seizure-induced spine changes with in vivo imaging, we performed a separate set of experiments to determine whether the acute spine loss observed within several hours after seizures persisted or recovered over a $24 \mathrm{~h}$ time period. In mice that were imaged sequentially for $24 \mathrm{~h}$ 
after stage 5 kainate seizures, the residual

spine loss that was observed several hours after the seizures on the first day showed no sign of recovery on the following day (Fig. 4). There was an $\sim 30 \%$ spine loss at both 4 and $24 \mathrm{~h}$ after seizure termination compared with the preseizure baseline $(n=300$ total spines from 26 dendrites from 4 mice). In contrast, control mice without seizures again show minimal changes in spine number $(<5 \%)$ over $24 \mathrm{~h}$.

\section{Kainate seizures cause acute activation of cofilin and depolymerization of F-actin}

We next investigated potential molecular mechanisms mediating the effects of kainate seizures on dendritic morphology. Actin is a major structural protein that can exist in a monomer form (G-actin) or a polymerized filamentous form (F-actin) and is highly concentrated in dendritic spines, forming complex filamentous networks that provide structural support for dendrites and dendritic spines (Matus et al., 1982; Capani et al., 2001). Because physiological activity may modulate actin networks to cause changes in dendritic structure and function (Kim and Lisman, 1999; Krucker et al., 2000; Fukazawa et al., 2003; Okamoto et al., 2004; Lin et al., 2005; Ouyang et al., 2005; Kramar et al., 2006), we examined the acute effects of kainate seizures on filamentous actin (Factin). Stage 5 kainate seizures for $30 \mathrm{~min}$ led to a significant decrease in F-actin in both the hippocampus and neocortex, as assayed by the rhodamine-phalloidin method (Fig. 5A,B). Compared with saline-injected controls, the decrease in F-actin labeling was seen immediately after termination of the seizures for at least $2 \mathrm{~h}$. Similar to results reported previously (Ouyang et al., 2005, 2007), doublelabeling experiments with MAP2 and PSD95 confirmed the localization of F-actin in dendrites and dendritic spines (data not shown). In a second assay of actin polymerization, insoluble (F-actin) and soluble (G-actin) fractions of actin were measured by Western blotting. Similar to the rhodamine-phalloidin results,

stage 5 kainate seizures caused a significant decrease in the ratio of F-actin to G-actin (Fig. 5C).

F-actin can be depolymerized by the regulatory actinbinding protein, cofilin. As cofilin is inactivated by phosphorylation, dephosphorylation of cofilin at the Ser3 residue leads to cofilin activation, which can trigger depolymerization of F-actin and may serve as a more sensitive marker of F-actin depolymerization. Thus, we tested whether kainate seizureinduced depolymerization of F-actin is related to a decrease in $10 \mu \mathrm{m}$.
Pre
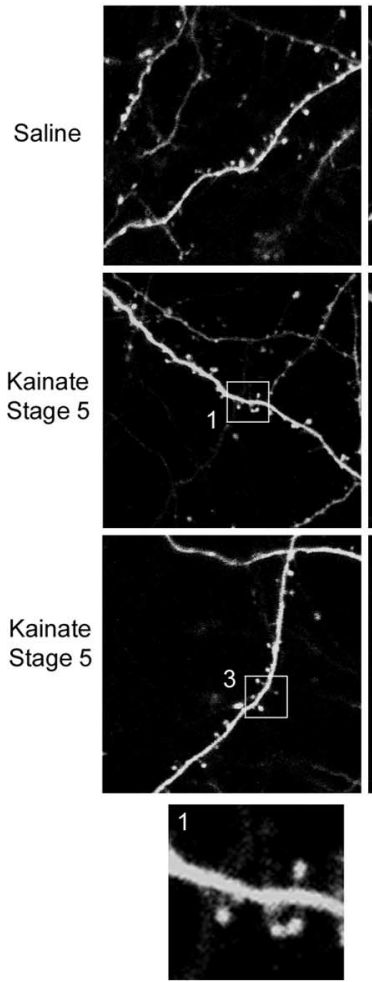

$0 \mathrm{hr}$
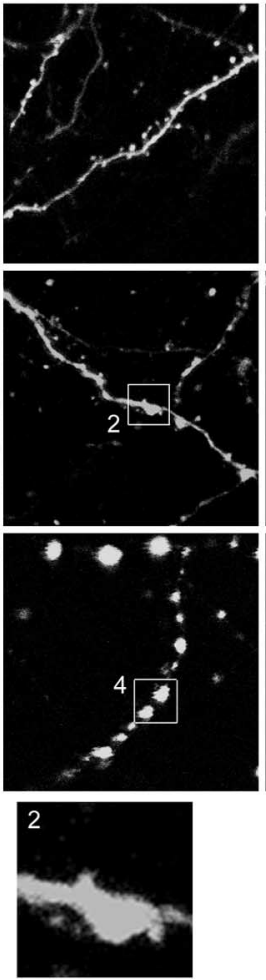

$1 \mathrm{hr}$
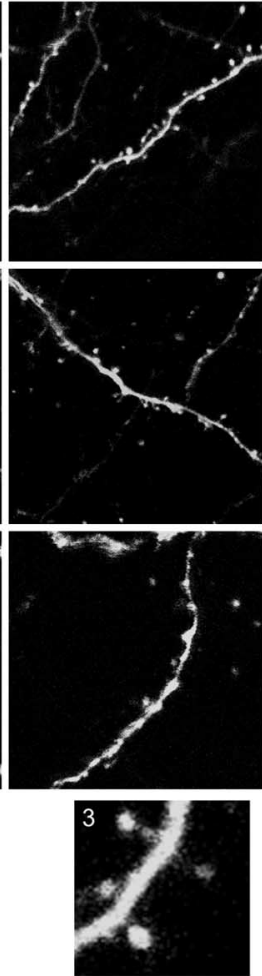

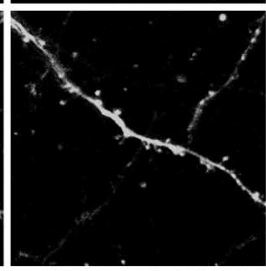

$2 \mathrm{~h}$
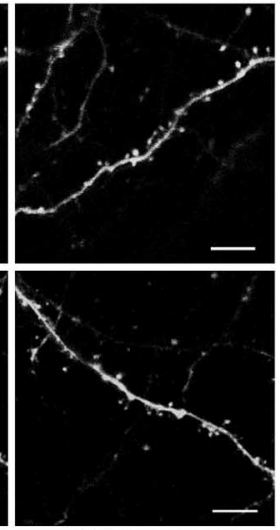

Figure 3. Representative images of dendritic changes in control conditions and after kainate seizures. After saline injection, mice always showed minimal changes in spines and no evidence of dendritic beading. In most cases, stage 4 kainate seizures also had minimal effects on dendritic spines or beading, although occasionally a mild beading with modest loss of spines was observed (not shown). By comparison, the majority of mice with stage 5 kainate seizures for 30 min exhibited immediate mild (middle sequence) or severe (bottom sequence) dendritic beading associated with a loss of spines (shown at higher power in excerpts). The dendritic beading usually recovered almost completely within $1-2 \mathrm{~h}$, but the recovery of spines was usually incomplete. Scale bar,

\section{Table 1. Kainate seizures cause a reversible dendritic beading in a stage-dependent manner}

\begin{tabular}{|c|c|c|c|c|}
\hline Seizure stage & Total dendrites & No beading & Mild beading & Severe beading \\
\hline \multicolumn{5}{|l|}{ Stage 4 seizure } \\
\hline Preseizure & 47 & 47 (100\%) & $0(0 \%)$ & $0(0 \%)$ \\
\hline $\mathrm{Oh}$ & 47 & 40 (85\%) & $7(15 \%)$ & $0(0 \%)$ \\
\hline $1 \mathrm{~h}$ & 46 & $39(85 \%)$ & $7(15 \%)$ & $0(0 \%)$ \\
\hline $2 \mathrm{~h}$ & 45 & $38(84 \%)$ & $7(16 \%)$ & $0(0 \%)$ \\
\hline $4 \mathrm{~h}$ & 14 & $12(86 \%)$ & $2(14 \%)$ & $0(0 \%)$ \\
\hline \multicolumn{5}{|l|}{ Stage 5 seizure } \\
\hline Preseizure & 49 & $49(100 \%)$ & $0(0 \%)$ & $0(0 \%)$ \\
\hline $\mathrm{Oh}$ & 49 & $10(20 \%)$ & $17(35 \%)$ & $22(45 \%)$ \\
\hline $1 \mathrm{~h}$ & 45 & $24(53 \%)$ & $15(33 \%)$ & $6(13 \%)$ \\
\hline $2 \mathrm{~h}$ & 45 & $29(64 \%)$ & $11(24 \%)$ & $5(11 \%)$ \\
\hline $4 \mathrm{~h}$ & 22 & $17(77 \%)$ & $3(14 \%)$ & $2(9 \%)$ \\
\hline
\end{tabular}

Mild beading, Visible beads with diameter of beads $<3 \times$ the diameter of the original dendrite with normal intervening segments of dendrite; severe beading, visible beading with diameter of beads $\geq 3 \times$ the diameter of the original dendrite without normal intervening segments of dendrite. Note that some mice were not imaged at $4 \mathrm{~h}$. By comparison, no beading was seen at all time points in all saline-injected control mice and mice treated with pentobarbital and kainate (no seizures). $p<0.001$ by $\chi^{2}$ test of independence for distribution of beading categories between no seizure, stage 4 , and stage 5 seizure groups at all time points, except $4 \mathrm{~h}$. 
A

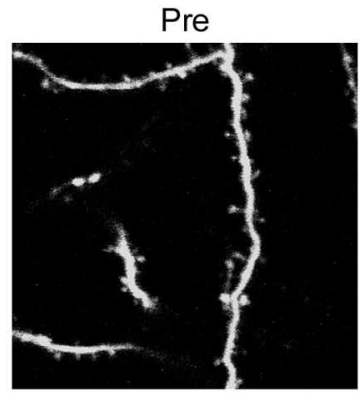

$4 \mathrm{hr}$

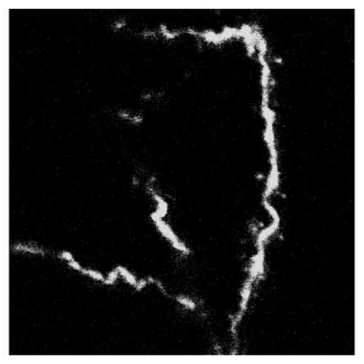

B

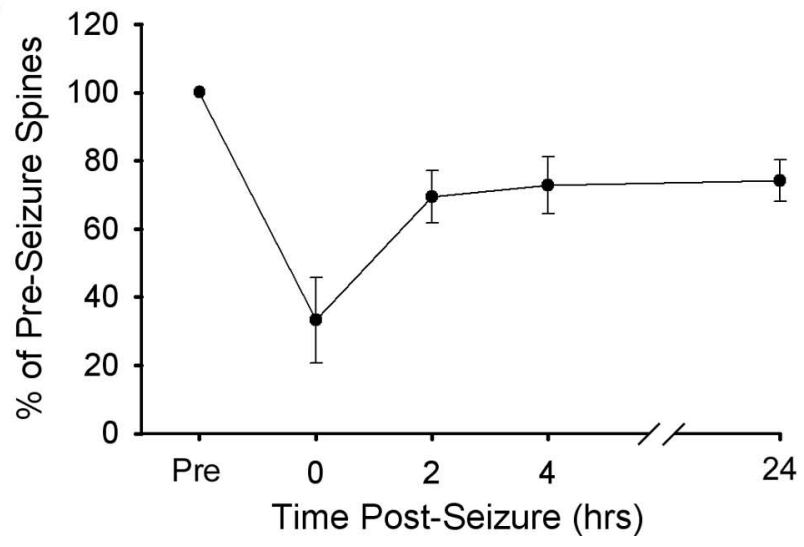

Figure 4. Dendritic spine loss persists for at least $24 \mathrm{~h}$ after kainate seizures. In separate experiments, a group of mice, which were monitored for acute changes in dendrites over several hours as in the previous experiments (Fig. 2), were reimaged $24 \mathrm{~h}$ after stage 5 kainate seizures. $A, B$, As described previously, dendritic beading developed and resolved within a couple hours. A partial recovery of spine loss also occurred with the resolution of dendritic beading, but a residual spine loss seen at $4 \mathrm{~h}$ after seizure persisted at $24 \mathrm{~h}(n=300$ total spines from 26 dendrites from 4 mice).

A calcineurin inhibitor antagonizes the effects of kainate seizures on cofilin activation and dendritic morphology

Cofilin and actin dynamics can be regulated by a number of upstream cellular signaling pathways, including a variety of phosphatases and kinases. In particular, the calcium-dependent phosphatase, calcineurin, is activated by seizures (Kurz et al., 2001, 2003) and may mediate regulation of cofilin activity by calcium (Wang et al., 2005). Thus, we used a calcineurin inhibitor to test whether calcineurin may be involved in the effects of kainate seizures on cofilin activation and dendritic morphology. First, we assessed whether calcineurin inhibitors may have direct effects on seizure properties or dendritic structure, which could confound interpretation of an observed, antagonist effect of these drugs on kainate seizure-induced dendritic injury. Consistent with previous studies (Moriwaki et al., 1998; Santos and Schwauwecker, 2003), pretreatment with the calcineurin inhibitor, FK506, $2 \mathrm{~h}$ before kainate injection had no effect on seizure severity/stage
(71\% vs $74 \%$ of mice achieved stage 5 seizures with FK506 vs saline pretreatment) or seizure latency $(19.9 \pm 2.6 \mathrm{~min}$ vs $20.4 \pm$ 2.5 min latency to stage 5 seizures for FK506 versus saline pretreatment, $p>0.5$ by $t$ test, $n=7$ mice per group). Furthermore, FK506 alone had no effect on dendritic structure, with no dendritic beading and $<5 \%$ spine turnover over $4 \mathrm{~h}$ (Fig. $7 B)(n=$ 167 total spines from 14 dendrites from 2 mice). However, FK506 administered $2 \mathrm{~h}$ before kainate significantly blocked the previously observed seizure-induced decrease in p-cofilin in both hippocampus and neocortex (Fig. 7A). In addition, FK506 pretreatment partially antagonized the effects of kainate seizures on dendritic beading and spine loss. After 30 min of stage 5 kainate seizure activity, mice pretreated with FK506 exhibited mild (7\%) or severe $(4 \%)$ beading in only $11 \%$ of cases, compared with $80 \%$ in untreated mice (Fig. 7B). Furthermore, FK506-treated mice only showed an $\sim 20 \%$ loss of overall spines immediately after the seizures ( $n=321$ total spines from 28 dendrites from 5 mice), compared with $\sim 60 \%$ spine loss in untreated mice (Fig. $7 B$ ). In contrast, FK506 administered immediately after 30 min of stage 5 kainate seizure activity had no significant protective effect against kainate seizure-induced dendritic beading and spine loss (Fig. $7 B)(n=311$ total spines from 29 dendrites from 3 mice), indicating that there may be a critical window during which FK506 must be present at the time of the seizure to have optimal effect. Overall, these results suggest that calcineurin may play a role in mediating the effects of kainate seizures on actin dynamics and spine morphology and demonstrate that calcineurin inhibitors may have therapeutic potential in limiting seizure-induced dendritic injury.

\section{Discussion}

Physiological and pathological activity-dependent modulation of dendritic structure and function is a subject of great scientific and clinical importance. A variety of studies have suggested that pathophysiological neuronal activity, such as seizures, can cause dendritic injury. However, much of this evidence has been derived from histopathological studies of fixed tissue from epilepsy patients or animal models [for review, see Swann et al. (2000) and Wong (2005)], which often have limitations related to potential confounding factors and inability to assay rapid dynamic changes. Newer imaging methods using time-lapse imaging of dendritic spines in vivo permit direct assessment of spine changes in individual neurons as a result of seizures. In this study, we demonstrate that kainate seizures can cause immediate dramatic changes in dendritic structure on the time scale of minutes. The live time-lapse imaging also directly revealed a rapid, dynamic evolution of dendritic abnormalities, not apparent in previous fixed tissue studies. In addition, we implicate actin depolymerization and calcineurin signaling as probable mechanisms for mediating these seizure-induced structural changes.

High-stage kainate seizures caused an immediate beading of dendrites and loss of spines, which displayed rapidly dynamic changes over a short time course. Although the dendritic beading, and to some extent, the loss of spines was reversible over a severalhour period, a plateau in the recovery of spines was observed starting $\sim 2 \mathrm{~h}$ after the seizures and persisting for at least $24 \mathrm{~h}$, suggesting that a residual, more permanent spine loss occurs. Although the purpose of the present study was to observe acute dynamic changes in spines immediately after seizures, future chronic in vivo imaging studies over days to weeks should be able to determine the longer-term time course of this spine loss. It is very likely that the spine loss seen in the present study is the initial phase of more chronic spine loss reported in other studies using 
conventional fixed tissue analysis (Scheibel et al., 1974; Isokawa and Levesque, 1991; Muller et al., 1993; Multani et al., 1994; Drakew et al., 1996; Isokawa, 1998; Jiang et al., 1998).

Although a variety of fixed tissue studies have found evidence of dendritic injury over a longer time scale, two recent acute in vivo imaging studies have found only modest effects of seizures on dendrites (Mizrahi et al., 2004; Rensing et al., 2005). Compared with these previous in vivo studies, which focused on more focal seizure activity in hippocampus or neocortex, the more robust effects of secondary generalized kainate seizures on dendrites in the present study likely reflect differences in seizure model, use of anesthesia, and the extent/severity of the seizures. In particular, kainate seizures likely activate more widespread cortical neuronal networks than the locally induced focal seizures in the previous studies. In the present study, there was a correlation between the "density" of electrographic seizure activity and the severity of dendritic injury comparing stage 4 and stage 5 seizures. Thus, it is likely that continuous status epilepticus may be necessary for the more overt structural changes. Although it is possible that systemic perturbations from seizures, rather than the electrical seizure activity itself, could also contribute to dendritic injury, mice did not display significant generalized convulsive activity ("stage 6") that is most often associated with systemic derangements, and blood gas analysis during the seizures was unremarkable, making this possibility unlikely. Finally, it is also possible that kainate could have a direct, toxic pharmacological effect causing dendritic injury, including activation of cell death mechanisms (Olney et al., 1979), but the use of C57BL/6 background mice minimizes this risk of kainate excitotoxicity, as reported by others (Schauwecker and Steward, 1997) and confirmed in the present study. Furthermore, the control group that was exposed to kainate but had seizures suppressed by pentobarbital showed no dendritic changes, indicating that seizure activity itself was responsible for the effects of kainate seizures on dendrites. Thus, our findings indicate that secondary generalized seizures can directly cause acute dendritic injury, independent of systemic factors or cell death.

The molecular mechanisms causing "nonlethal" seizure-induced dendritic injury are largely unexplored. In contrast, there is an expanding literature demonstrating that more physiological forms of activity, such as tetanic stimulation to induce long-term potentiation (LTP), may cause structural and functional changes in dendrites as a result of regulation of the actin cytoskeleton. As physiological activation of neurons

B

C
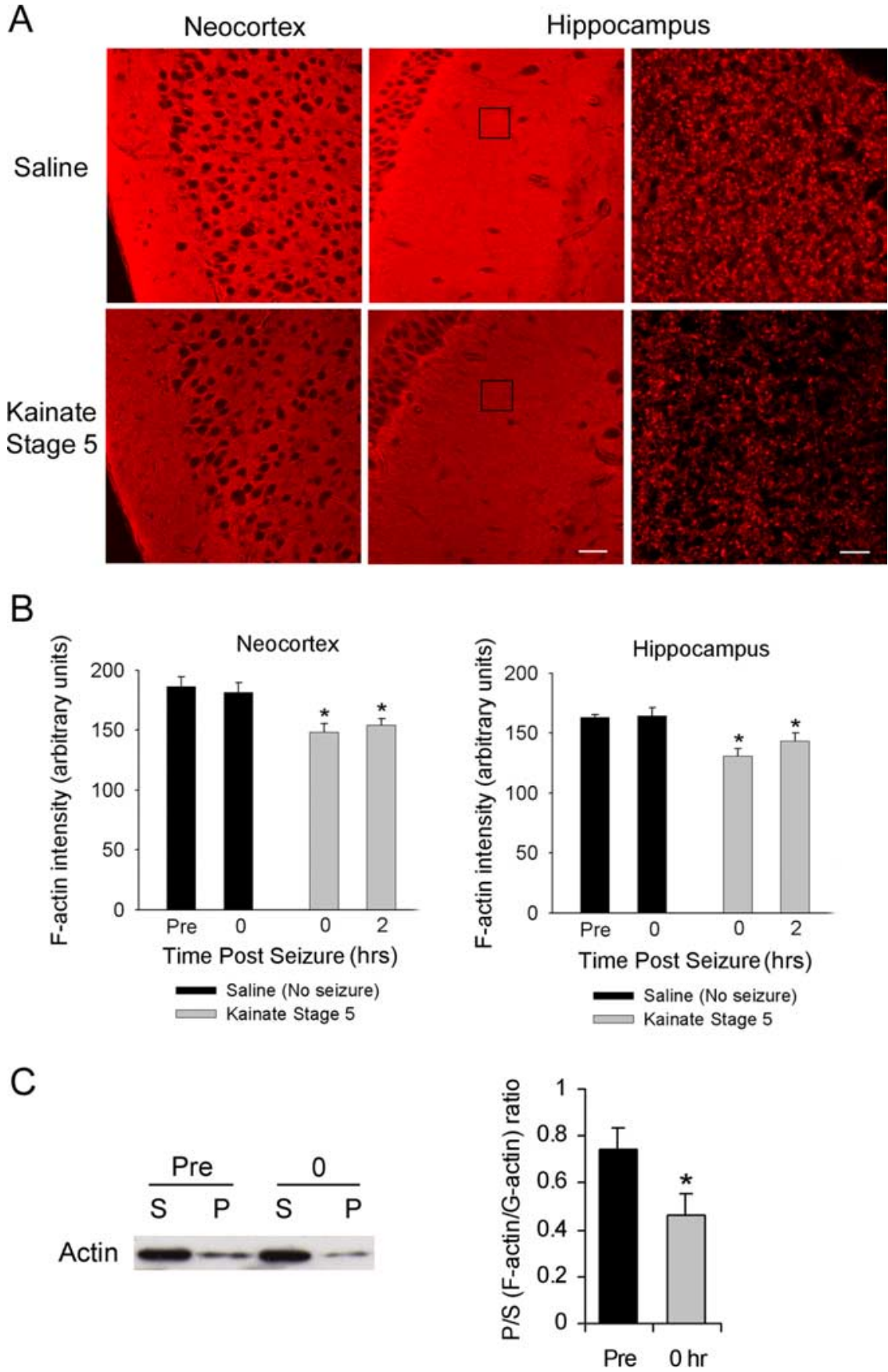

Figure 5. Kainate seizures cause acute depolymerization of $\mathrm{F}$-actin. $A$, Representative images of $\mathrm{F}$-actin labeling, assayed by the rhodamine-phalloidin method, are shown in the neocortex (left) and stratum radiatum of CA1 region (middle) of a control (saline-injected) mouse and a mouse after 30 min of stage 5 kainate seizure activity. Scale bar, $40 \mu \mathrm{m}$. On higher magnification (right), punctate labeling is observed, typical of dendritic spine localization of F-actin. Scale bar, $5 \mu \mathrm{m}$. B, Summarized data for all experiments show a decrease in F-actin rhodamine-phalloidin labeling in neocortex and hippocampus for $0-2 \mathrm{~h}$ after kainate seizures. ${ }^{*} p<0.01$ by one-way ANOVA ( $n=6-8$ sections/mouse, $n=5$ mice per saline groups, $n=8$ mice per kainate groups). $C$, In a second assay of actin polymerization, Western blotting for actin was performed on fractions of soluble actin (G-actin) in supernatant $(S)$ and insoluble actin ( $F$-actin) in the pellet $(P)$ separated by centrifugation from homogenized cortex. A representative Western blot from one experiment is shown. Supernatant and pellet fractions from each sample were proportionally loaded for all conditions/experiments, and the ratio of F-actin to $\mathrm{G}$-actin was calculated. Summarized data for all experiments show that stage 5 kainate seizures for 30 min caused an immediate decrease in the ratio of $\mathrm{F}$-actin to $\mathrm{G}$-actin, confirming that kainate seizures cause depolymerization of F-actin. ${ }^{*} p<0.05$ by $t$ test ( $n=4$ mice per group).

may lead to either increases (Fukazawa et al., 2003; Okamoto et al., 2004; Lin et al., 2005; Ouyang et al., 2005; Kramar et al., 2006) or decreases (Kim and Lisman, 1999; Shen and Meyer, 1999; Ouyang et al., 2005) in actin polymerization, we have recently 

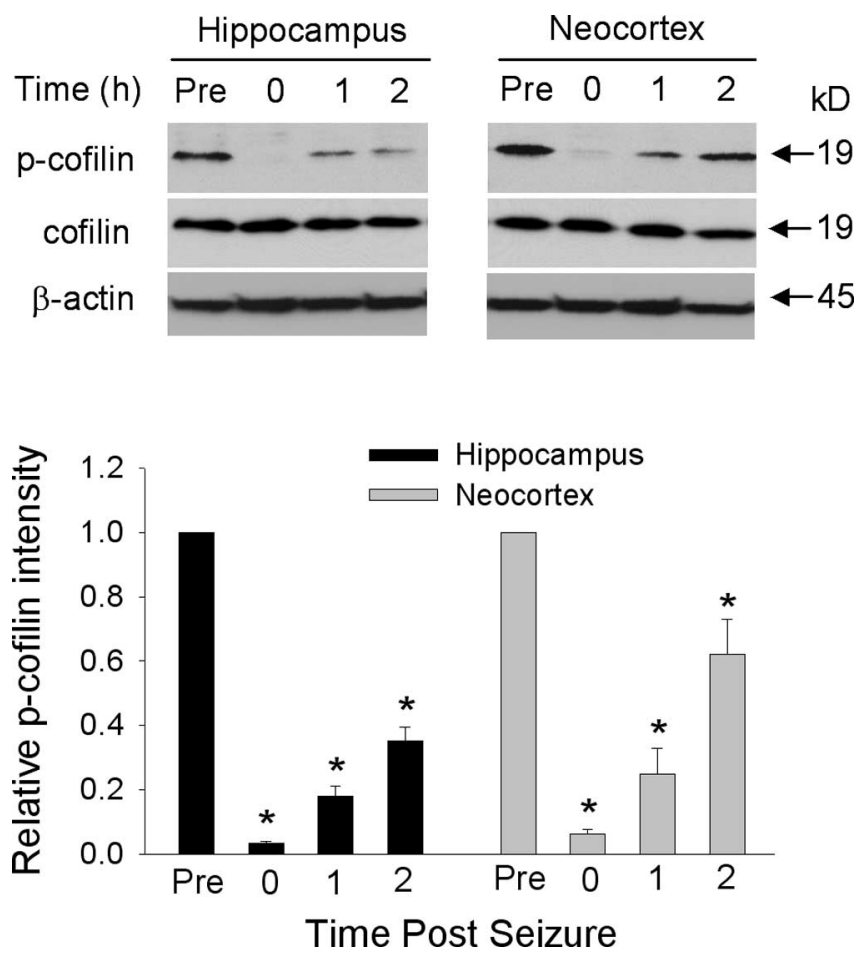

Figure 6. Kainate seizures cause acute activation of cofilin. Top, Phosphorylated cofilin (pcofilin), total cofilin, and actin were assayed by Western blotting of hippocampus and neocortex from control (saline-injected) mice and mice after 30 min of stage 5 kainate seizure activity. A representative Western blot from one experiment is shown. Bottom, Summarized data for all experiments show a dramatic decrease in relative protein expression of $\mathrm{p}$-cofilin after kainate seizures. The ratio of $\mathrm{p}$-cofilin to total cofilin was calculated for each condition/experiment and normalized to the preseizure control. ${ }^{*} p<0.001$ by one-way ANOVA ( $n=12$ mice per group).

reported that conditions favoring LTP induction may cause a biphasic response, involving an initial transient decrease in F-actin in dendritic spines followed by a longer-term increase in actin polymerization (Ouyang et al., 2005). In LTP, we hypothesize that the initial phase of actin depolymerization may allow for plasticity and motility of dendritic structure or function, whereas subsequent polymerization of F-actin could lead to long-term stabilization or consolidation of dendritic changes. By comparison, pathological neuronal activation, such as with seizures, might disrupt this finely regulated, dynamic system of dendritic actin networks. In support of this idea, we have recently shown that hippocampal seizures induced by 4-AP lead to moderate activation of cofilin, a major actin-depolymerizing factor, and associated depolymerization of F-actin, although these changes were not necessarily associated with any overt structural changes in dendrites, perhaps because of the relatively mild nature of the seizures (Ouyang et al., 2007). In the present study, we demonstrate that kainate seizures cause a stronger activation of cofilin and depolymerization of F-actin, which is associated with dramatic structural changes in dendrites and is, at least in part, mediated by the calcium-activated phosphatase, calcineurin. Thus, there likely exists a spectrum of physiological and pathological activity that can regulate similar actin-based mechanisms to cause both normal synaptic plasticity under physiological situations and abnormal dendritic injury during extreme conditions. Because calcineurin can also regulate a number of other cellular pathways, future studies are required to determine all the specific intracellular signaling and mechanistic elements involved in mediating the effects of seizures on dendritic structure. The role of other critical triggering or modulatory factors associated with seizures, such as elevated extracellular potassium, glutamate release, and local hypoxia, also needs to be explored.

In addition to the importance of understanding activitydependent regulation of actin dynamics and dendritic structure on a mechanistic level, the findings from this study may have important clinical and therapeutic implications. Seizure-induced brain injury may contribute to a number of behavioral, cognitive, and neuropsychiatric deficits commonly seen in epilepsy patients (Dodrill, 2002; Elger et al., 2004). Although seizure-induced neuronal death has been widely documented and studied, especially in animal models, many epilepsy patients have no overt evidence of neuronal death, at least on structural brain imaging, despite suffering from these neurological comorbidities. Thus, understanding "nonlethal" mechanisms of seizure-induced brain injury, such as changes in dendritic structure and function, may have more widely applicable clinical relevance and may ultimately lead to novel therapeutic strategies either for treating seizures or preventing neurocognitive deficits in epilepsy. Whereas most drugs for epilepsy have targeted neurotransmitter receptors and ion channels, an innovative therapeutic approach would be to modulate actin-based spine motility in an activity-dependent manner. In the present study, we demonstrate the potential therapeutic benefit of calcineurin inhibitors, such as FK506, in limiting seizure-induced dendritic injury. It is possible that the protective effect of FK506 could actually be caused by a nonspecific action of FK506 in reducing neuronal excitability or seizures, not by a specific effect on mechanisms of seizure-induced dendritic injury. However, consistent with previous studies (Moriwaki et al., 1998; Santos and Schwauwecker, 2003), FK506 did not alter kainate seizure latency or severity, indicating that FK506 did not directly alter seizures per se. Furthermore, FK506 alone had no obvious effect on dendritic morphology. Thus, FK506 most likely has specific protective effects against mechanisms of seizureinduced dendritic injury via direct calcineurin-mediated modulation of actin. One limitation of the potential therapeutic applications of this finding is that our data suggest that the drug needs to be administered prophylactically before the onset of a seizure to be most effective. Future research might find that selective stabilization of the dendritic actin cytoskeleton during or possibly after seizures by other drugs that directly regulate actin polymerization (Ackermann and Matus, 2003) could be even more effective in preventing seizure-induced spine changes and potentially reducing resultant neurocognitive deficits. Thus, better insights into the mechanisms of modulation of actin-based spine dynamics by seizures could have significant impact in reducing the long-term negative consequences of epilepsy.

\section{References}

Ackermann M, Matus A (2003) Activity-induced targeting of profilin and stabilization of dendritic spine morphology. Nat Neurosci 6:1194-1200.

Ben-Ari Y, Cossart R (2000) Kainate, a double agent that generates seizures: two decades of progress. Trends Neurosci 23:580-587.

Capani F, Martone ME, Deerinck TJ, Ellisman MH (2001) Selective localization of high concentrations of F-actin in subpopulations of dendritic spines in rat central nervous system: a three-dimensional electron microscopic study. J Comp Neurol 435:156-170.

Dodrill CB (2002) Progressive cognitive decline in adolescents and adults with epilepsy. Prog Brain Res 135:399-407.

Drakew A, Muller M, Gahwiler BH, Thompson SM, Frotscher M (1996) Spine loss in experimental epilepsy: quantitative light and electron microscopic analysis of intracellularly stained CA3 pyramidal cells in hippocampal slice cultures. Neuroscience 70:31-45.

Elger CE, Helmstaedter C, Kurthen M (2004) Chronic epilepsy and cognition. Lancet Neurology 3:663-672.

Feng G, Mellor RH, Bernstein M, Keller-Peck C, Nguyen QT, Wallace M, 
A

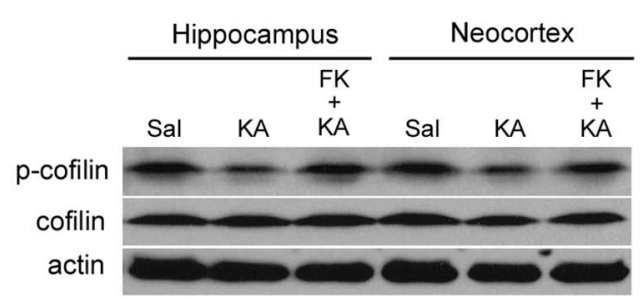

B

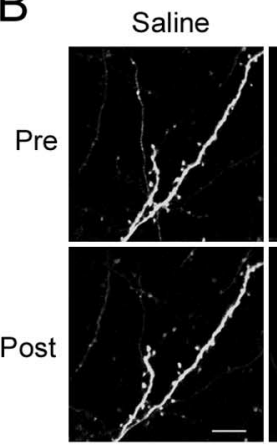

FK

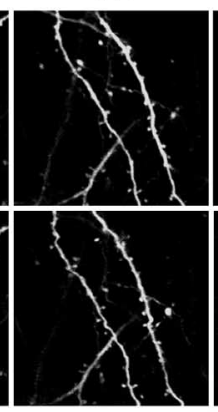

KA

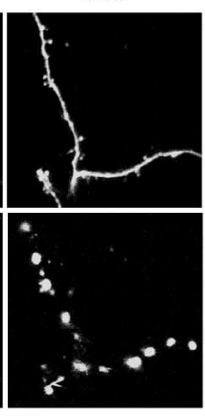

$\mathrm{FK}+\mathrm{KA}$

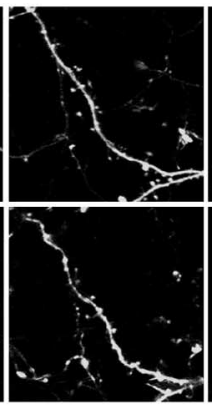

$\mathrm{KA}+\mathrm{FK}$
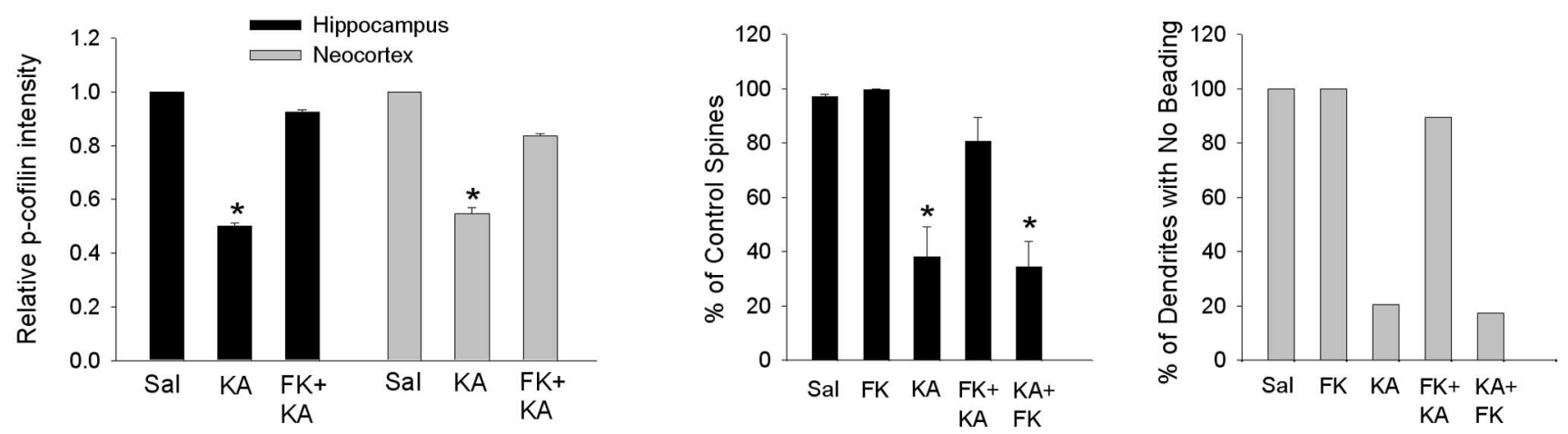

Figure 7. A calcineurin inhibitor antagonizes the effects of kainate seizures on cofilin activation and dendritic morphology. A, Phosphorylated cofilin (p-cofilin), total cofilin, and actin were assayed by Western blotting of hippocampus and neocortex from saline-injected control mice (Sal), mice immediately after 30 min of stage 5 kainate seizure activity (KA), and mice immediately after 30 min of stage 5 kainate seizure activity pretreated with FK506 (FK + KA). Pretreatment with FK506 blocked the seizure-induced decrease in p-cofilin in both hippocampus and neocortex. ${ }^{*} p<$ 0.01 by one-way ANOVA ( $n=6$ mice per group). $\boldsymbol{B}$, FK506 partially antagonized the morphological effects of kainate seizures on neocortical dendrites. FK506 alone had no effect on dendritic morphology (FK; $n=167$ total spines from 14 dendrites from 2 mice), similar to saline-injected controls (Sal; $n=400$ total spines from 33 dendrites from 5 mice). As before, stage 5 kainate seizures for 30 min usually caused immediate dendritic beading and loss of spines (KA; $n=531$ total spines from 49 dendrites from 9 mice). Pretreatment with FK506 (FK $+K A ; n=321$ total spines from 28 dendrites from 5 mice) significantly reduced the kainate seizure-induced spine loss and dendritic beading, whereas FK506 administered immediately after 30 min of stage 5 seizure activity (KA + FK; $n=311$ total spines from 29 dendrites from 3 mice) had no effect on the seizure-induced dendritic changes. The graphs show data from the first, immediate postseizure images, but similar differences between the groups were seen at all time points measured at 1,2 , and $4 \mathrm{~h}$ after seizure. ${ }^{*} p<0.01$ by one-way ANOVA with posttests comparing KA and KA + FK groups to all other groups. Scale bar, $10 \mu \mathrm{m}$.

Nerbonne JM, Lichtman JW, Sanes JR (2000) Imaging neuronal subsets in transgenic mice expressing multiple spectral variants of GFP. Neuron 28:41-51.

Fukazawa Y, Saitoh Y, Ozawa F, Ohta Y, Mizuno K, Inokuchi K (2003) Hippocampal LTP is accompanied by enhanced F-actin content with the dendritic spine that is essential for late LTP maintenance in vivo. Neuron 38:447-460.

Grutzendler J, Kasthuri N, Gan WB (2002) Long-term dendritic spine stability in the adult cortex. Nature 420:812-816.

Gu YY, Zhang HY, Zhang HJ, Li SY, Ni JH, Jia HT (2006) 8-Chloroadenosine inhibits growth at least partly by interfering with actin polymerization in cultured human lung cancer cells. Biochem Pharmacol 72:541-550.

Holtmaat AJ, Trachtenberg JT, Wilbrecht L, Shepherd GM, Zhang X, Knott GW, Svoboda K (2005) Transient and persistent dendritic spines in the neocortex in vivo. Neuron 45:279-291.

Isokawa M (1998) Remodeling dendritic spines in the rat pilocarpine model of temporal lobe epilepsy. Neurosci Lett 258:73-76.

Isokawa M, Levesque MF (1991) Increased NMDA responses and dendritic degeneration in human epileptic hippocampal neurons in slices. Neurosci Lett 132:212-216.

Jiang M, Lee CL, Smith KL, Swann JW (1998) Spine loss and other persistent alterations of hippocampal pyramidal cell dendrites in a model of earlyonset epilepsy. J Neurosci 18:8356-8368.

Kim CH, Lisman JE (1999) A role of actin filament in synaptic transmission and long-term potentiation. J Neurosci 19:4314-4324.

Kramar EA, Lin B, Rex CS, Gall CM, Lynch G (2006) Integrin-driven actin polymerization consolidates long-term potentiation. Proc Natl Acad Sci USA 103:5579-5584.

Krucker T, Siggins GR, Halpain S (2000) Dynamic actin filaments are re- quired for stable long-term potentiation (LTP) in area CA1 of the hippocampus. Proc Natl Acad Sci USA 97:6856-6861.

Kurz JE, Sheets D, Parsons JT, Rana A, Delorenzo RJ, Churn SB (2001) A significant increase in both basal and maximal calcineurin activity in the rat pilocarpine model of status epilepticus. J Neurochem 78:304-315.

Kurz JE, Rana A, Parsons JT, Churn SB (2003) Status epilepticus-induced changes in the subcellular distribution and activity of calcineurin in rat forebrain. Neurobiol Dis 14:483-493.

Leite JP, Garcia-Cairasco N, Cavalheiro EA (2002) New insights from the use of pilocarpine and kainate models. Epilepsy Res 50:93-103.

Lendvai B, Stern EA, Chen B, Svoboda K (2000) Experience-dependent plasticity of dendritic spines in the developing rat barrel cortex in vivo. Nature 404:876-881.

Lin B, Kramar EA, Bi X, Brucher FA, Gall CM, Lynch G (2005) Theta stimulation polymerizes actin in dendritic spines of hippocampus. J Neurosci 25:2062-2069.

Matus A, Ackermann M, Pehling G, Byers HR, Fujiwara K (1982) High actin concentrations in brain dendritic spines and postsynaptic densities. Proc Natl Acad Sci USA 79:7590-7594.

Mizrahi A, Crowley JC, Shtoyerman E, Katz LC (2004) High-resolution in vivo imaging of hippocampal dendrites and spines. J Neurosci 24:3147-3151.

Moriwaki A, Lu YF, Tomizawa K, Matsui H (1998) An immunosuppressant, FK506, protects against neuronal dysfunction and death but has no effect on electrographic and behavioral activities induced by systemic kainate. Neurosci 86:855-865.

Muller M, Gahwiler BH, Rietschin L, Thompson SM (1993) Reversible loss of dendritic spines and altered excitability after chronic epilepsy in hippocampal slice cultures. Proc Natl Acad Sci USA 90:257-261.

Multani P, Myers RH, Blume HW, Schomer DL, Sotrel A (1994) Neocorti- 
cal dendritic pathology in human partial epilepsy: a quantitative Golgi study. Epilepsia 35:728-736.

Okamoto K, Nagai T, Miyawaki A, Hayashi Y (2004) Rapid and persistent modulation of actin dynamics regulates postsynaptic reorganization underlying bidirectional plasticity. Nat Neurosci 7:1104-1112.

Olney JW, Fuller T, deGubareff T (1979) Acute dendrotoxic changes in the hippocampus of kainate treated rats. Brain Res 176:91-100.

Olney JW, deGubareff T, Sloviter RS (1983) "Epileptic" brain damage in rats induced by sustained electrical stimulation of the perforant path. II. Ultrastructural analysis of acute hippocampal pathology. Brain Res Bull 10:699-712.

Ouyang Y, Wong M, Capani F, Rensing N, Lee CS, Liu Q, Martone ME, Wu J, Ellisman MH, Yamada K, Choi DW (2005) A transient decrease in F-actin may be necessary for translocation of proteins into dendritic spines. Eur J Neurosci 22:2995-3005.

Ouyang Y, Yang XF, Hu XY, Erbayat-Altay E, Zeng LH, Lee JM, Wong M (2007) Hippocampal seizures cause depolymerization of filamentous actin in neurons independent of acute morphological changes. Brain Res 1143:238-246.

Racine RJ (1972) Modification of seizure activity by electrical stimulation: II. Motor seizure. Electroencephalogr Clin Neurophysiol 32:281-294.

Rensing NR, Ouyang Y, Yang XF, Yamada KA, Rothman SM, Wong M (2005) In vivo imaging of dendritic spines during electrographic seizures. Ann Neurol 58:888-898.

Santos JB, Schwauwecker PE (2003) Protection provided by cyclosporin A against excitotoxic neuronal death is genotype dependent. Epilepsia 44:995-1002.

Schauwecker PE, Steward O (1997) Genetic determinants of susceptibility to excitotoxic cell death: implications for gene targeting approaches. Proc Natl Acad Sci USA 94:4103-4108.

Scheibel ME, Crandall PH, Scheibel AB (1974) The hippocampal-dentate complex in temporal lobe epilepsy. Epilepsia 15:55-80.

Schmued LC, Hopkins KJ (2000) Fluoro-Jade B: a high affinity fluorescent marker for the localization of neuronal degeneration. Brain Res 874:123-130.

Shen M, Meyer T (1999) Dynamic control of CaMKII translocation and localization in hippocampal neurons by NMDA receptor stimulation. Science 284:162-166.

Swann JW, Al-Noori S, Jiang M, Lee CL (2000) Spine loss and other dendritic abnormalities in epilepsy. Hippocampus 10:617-625.

Trachtenberg JT, Chen BE, Knott GW, Feng G, Sanes JR, Welker E, Svoboda $\mathrm{K}$ (2002) Long-term in vivo imaging of experience-dependent synaptic plasticity in adult cortex. Nature 420:788-794.

Wang Y, Shibasaki F, Mizuno K (2005) Calcium signal-induced cofilin dephosphorylation is mediated by Slingshot via calcineurin. J Biol Chem 280:12683-12689.

Wong M (2005) Modulation of dendritic spines in epilepsy: cellular mechanisms and functional implications. Epilepsy Behav 7:569-577.

Wong M, Wozniak M, Yamada KA (2003) An animal model of generalized nonconvulsive status epilepticus: immediate characteristics and longterm effects. Exp Neurol 183:87-99. 